\title{
Mechanism study and numerical simulation of Uranium nitriding induced by high energy laser
}

\author{
Yuan Zhu, Jingjing Xu, Yanwen Qi, Shengpeng Li, Hui Zhao \\ School of Mechatronic Engineering and Automation, Shanghai University, Shanghai 200072, China
}

\begin{abstract}
The gradients of interfacial tension induced by local heating led to Marangoni convection, which had a significant effect on surface formation and the process of mass transport in the laser nitriding of uranium. An experimental observation of the underlying processes was very difficult. In present study, the Marangoni convection was considered and the computational fluid dynamic (CFD) analysis technique of FLUENT program was performed to determine the physical processes such as heat transfer and mass transport. The progress of gas-liquid falling film desorption was presented by combining phase-change model with fluid volume function (VOF) model. The time-dependent distribution of the temperature had been derived. Moreover, the concentration and distribution of nitrogen across the laser spot are calculated. The simulation results matched with the experimental data. The numerical resolution method provided a better approach to know the physical processes and dependencies of the coating formation.
\end{abstract}

\section{Introduction}

Since uranium metal readily corrodes under the ambient environment, many technologies were developed to improve its corrosion resistance $[1,2]$, where nitriding and carbonation of the surface are well known for improving the tribological properties of different metal compounds. Pulsed laser nitriding has shown that the metal-nitride layer produced by a high-power pulsed laser greatly improves the surface hardness and the corrosion resistance [3]. Due to its high power and flexibility in its temporal shaping, this type of laser could be the appropriate tool. The nitrogen transport and corresponding coating properties are determined by the time of treatment, for which diffusion will be assisted by the Marangoni convection for extended time regimes. Because time-dependent occurrences were inaccessible in experiment, many researches have done using numerical simulation method. Daniel Höche et al [4,5] established the N-Ti two-dimensional (2-D) model in Comsol, which was under the influence of the Marangoni effect on the formation of molten pool. Fuzhen Xuan [6] established the coupling model of the transient temperature field and the concentration field. The model was based on the unsteady state Fourier heat conduction equation and the Fick law of modified diffusion equation, the effect of a moving heat source on the formation of temperature gradient, which lead to nitrogen diffusion in titanium, was taken into account. However, as of now, there has been no in depth research conducted on the nitriding of actinide metal. Meanwhile, in the previous study, the factors in the process of laser nitriding for different problems are not considered comprehensively. In this paper, the effects of Marangoni convection, temperature gradient and fluid flow are investigated using the computational fluid dynamic analysis technique of FLUENT. The transient N-U twodimensional model is established, and the two-film theory model is applied. Absorption of nitrogen into a laminar falling film on the interface is presented using the volume of fluid (VOF) model. Consequently, the mechanism of heat and mass transfer were established, and the simulation results agree with the experimental results. In addition, the relationship between the parameters and the properties of nitride coating was revealed. This finally provides references for theory analysis and allows an optimization of the coating synthesis.

\section{Numerical Simulation Model}

\subsection{Physical Model}

Uranium sheets (20 $\mathrm{mm}$ in diameter, $2 \mathrm{~mm}$ in thickness) were placed in a chamber evacuated and filled with $99.99 \%$ pure nitrogen gas. In order to treat extended areas, the vacuum chamber was placed on a twodimensional monitored linear stage. The laser scanned along the $\mathrm{Y}$ direction with speeds ranging from 0.1 to $1.28 \mathrm{~mm} / \mathrm{s}$. After scanning a line in the $\mathrm{Z}$ direction, the vacuum chamber moved $1 \mathrm{~mm}$ in $X$ direction [7,8]. In Daniel Höche and Sven Müller's research, a feasible two-dimensional simplified computational model was constructed, and the simulation results matched experimental results very well. The scan velocity, which the model is based on, is much lower than the melt flow velocity. Here, we assumed that the present case

\footnotetext{
* Corresponding author: zhuyuan1993@163.com
} 
matched with Daniel Höche's, so a transient and twodimensional model (plane XY) is used to describe the nitriding of uranium. The $5 \times 4 \mathrm{~mm} 2$ simplified plane model was established in the simulation.

Figure 1 shows the different domains $\left(\mathrm{N}_{2}\right.$ and uranium) and several boundaries. The temperature condition of the thin uranium sheet is given by $T_{0}=300 \mathrm{~K}$. The PLANE13 2-D 4-node quadrilateral mesh was applied in simulation, which contains 50000 elements with 50451 nodes. Elements were linear in this case of heat transfer and diffusion. At the boundaries of the model (walls), the velocity was set to be zero (no slip). On the gaseous side (outlet), a pressure-outlet condition was employed. The initial temperature was set to $300 \mathrm{~K}$, the equivalent laser heat source is setted at the surface of uranium.

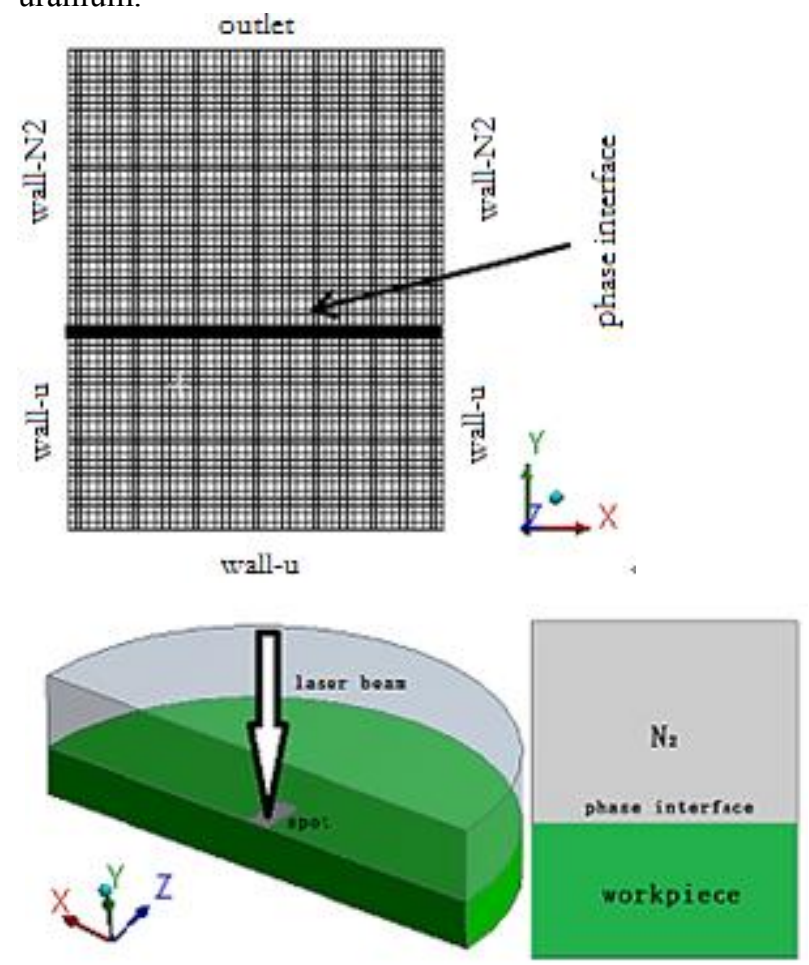

Fig. 1. Geometry and Grid

The laser beam was focused on the sample using a convex lens with spot size of $1 \times 2 \mathrm{~mm}^{2}$, which was a temporal pulse structure in a continuous wave mode. The beam was approximately $1 \mathrm{~mm}$ in width at cross-section.

In the simulation, in order to better use numerical simulation methods to study nitriding process, the millisecond time of uranium treatment is applied. The form of the laser-pulse intensity $I$ adopted in our simulation will be taken as "rectangle":

$$
\left\{\begin{array}{l}
I(x, t)=\left(1-R_{s, l}\right) \frac{p}{A}, 0 \leq t \leq \tau,-0.0005 \leq x \leq 0.0005 \\
I(x, t)=0, \tau \leq t
\end{array}\right.
$$

\subsection{Heating Transport}

The effect of atomic fission was ignored because of the short interaction time and insufficient energy of the laser. We made use of the heat flow equation in twodimensional (2-D) space to describe the heat flow. The mathematical description of phase changes is a difficulty formally known as the Stefan problem. To solve the problem the smoothed Heaviside function $(T, B)$ was used. $B$ is the width of the temperature region where $(T$, $B$ ) changes from 0 to 1 and was assumed to be $100 \mathrm{~K}$.

$$
G(T)=G_{\text {sol }}-\left(G_{\text {sol }}+G_{\text {liq }}\right) \Theta(T, B)
$$

Here, the energy conservation equation is established in the solid phase and liquid phase region, respectively, the temperature $T$ as the only variable.

$$
\beta(T) C_{p}(T) \frac{\partial T}{\partial t}=\nabla(\kappa(T) \nabla T)+S-v_{r} \rho(T) C_{p}(T) \nabla T
$$

Where $T, c_{\mathrm{p}}(T)$, and $\kappa(T)$ are, respectively, temperature, specific heat and thermal conductivity and $S$ is heat source term given by Eq.(4). The quantities $c, \rho$, and $\kappa$ will be subsequently assumed to be temperature and space independent. $S$ can be written using UDF in Fluent.

Where $T_{0}$ is the ambient temperature $\left(T_{0}=300 \mathrm{~K}\right)$.

Table 1 and table 2 give the material physical properties used in the present modeling work. Additionally, the external absorption parameter $\alpha_{\text {ext }}$ was defined to account for heat losses due to the optics.

Table 1. Physical Properties of uranium Used in Simulations

\begin{tabular}{|c|c|c|}
\hline Melting temperature/K & $T_{\mathrm{L}}$ & 1405 \\
\hline $\begin{array}{c}\text { Boiling } \\
\text { temperature at } 1 \text { atm/K }\end{array}$ & $T_{\mathrm{b}}$ & 4091 \\
\hline $\begin{array}{c}\text { Latent heat of fusion } / \text { vaporization } \\
/\left(\mathrm{kJ} \cdot \mathrm{kg}^{-1}\right)\end{array}$ & $L_{\mathrm{m}} / L_{\mathrm{ev}}$ & $\begin{array}{c}1159.2 / \\
38364\end{array}$ \\
\hline $\begin{array}{c}\text { Solid/ Liquid phase density } \\
/\left(\mathrm{kg} \cdot \mathrm{m}^{-3}\right)\end{array}$ & $\rho_{\mathrm{s}} / \rho_{\mathrm{l}}$ & $\begin{array}{c}19100 / \\
17300\end{array}$ \\
\hline $\begin{array}{c}\text { Molar mass } \\
/\left(\mathrm{g} \cdot \mathrm{mol}^{-1}\right)\end{array}$ & $M_{\mathrm{u}}$ & 238 \\
\hline $\begin{array}{c}\text { Viscosity at } 1405 \mathrm{~K} \\
/ \mathrm{mpa}^{\mathrm{s}} \mathrm{s}\end{array}$ & $\eta_{\mathrm{u}}$ & 6.55 \\
\hline $\begin{array}{c}\text { Surface tension } \\
/\left(\mathrm{N} \cdot \mathrm{m}^{-1}\right)\end{array}$ & $\sigma$ & 1.55 \\
\hline $\begin{array}{c}\text { Gradient of surface tension/ } \\
\left(\mathrm{N} \cdot \mathrm{m}^{-1} \cdot \mathrm{K}^{-1}\right)\end{array}$ & $\partial \sigma / \partial T$ & $\begin{array}{c}-1.4 \times \\
10^{-4}\end{array}$ \\
\hline
\end{tabular}

Table 2. Physical Properties of Nitrogen Used in Simulations.

\begin{tabular}{|c|c|c|}
\hline Density $/\left(\mathrm{kg} \cdot \mathrm{m}^{-3}\right)$ & $\rho_{N_{2}}$ & 1.138 \\
\hline $\begin{array}{c}\text { Thermal conductivity/ } \\
\left(\mathrm{W} \cdot \mathrm{m}^{-1} \cdot \mathrm{K}^{-1}\right)\end{array}$ & $\kappa_{N_{2}}$ & 0.026 \\
\hline $\begin{array}{c}\text { Specific heat ratio/ } \\
\left(\mathrm{J} \cdot \mathrm{kg}^{-1} \cdot \mathrm{K}^{-1}\right)\end{array}$ & $c_{P_{2}}$ & 1040 \\
\hline Viscosity $/ \mu \mathrm{pa} \cdot \mathrm{s}$ & $\mu_{N_{2}}$ & 3.2 \\
\hline
\end{tabular}

\subsection{Mass Transport}

With the laser irradiated, nitrogen gas absorbed energy, forming nitrogen atoms, which were diffuser into a molten pool. This process occurred at the gas-liquid interface, and was in accord with the falling film desorption. Interphase mass transport always exists if only the bulk concentration of gas-liquid does not reach equilibrium. The process consists of a gas-liquid twophase stratified flow. In order to model surface tracking, 
we applied the VOF interface tracking technology. VOF can accurately simulate the unstable of liquid film at free surface, and is especially suitable for flow filed, which has obvious interface.

The process includes the mass conservation Eq. (5), momentum conservation Eq. (6).

$$
\begin{aligned}
& \frac{\partial}{\partial t}(\rho)+\nabla(\rho v)=0 \\
& \frac{\partial}{\partial t}(\rho \stackrel{\mathrm{r}}{\mu})+\nabla(\rho \stackrel{\mathrm{rr}}{\mathrm{rr}})=-\nabla p+\nabla\left[\mu\left(\nabla \stackrel{\mathrm{r}}{v}+\stackrel{\mathrm{r}}{v}^{T}\right)\right]+\rho \stackrel{\mathrm{r}}{g}+\stackrel{\mathrm{r}}{F}
\end{aligned}
$$
the sum of the different phase density, and $\vec{v}$ the momentum source.

When the molten pool was formed, there was obvious gas-liquid interface; combined with double film theory and penetration theory, mass transport equation is as follows:

$$
S_{G L}^{k}=\frac{\partial \alpha_{q} \rho_{q} w_{q}^{k}}{\partial t}+\nabla\left(\alpha_{q} \rho_{q} \underset{\mu}{\mu} w_{q}^{k}-\alpha_{q} \Gamma_{q}^{k} \nabla w_{q}^{k}\right) ; q=L, G ; k=1, \ldots ., r
$$

The mass transfer source $S_{G L}^{k}$, which is the speed of species $k$ in one phase transport to another phase ; $w_{q}^{k k}$ is the mass fraction of species $k$ in phase $q ; \Gamma_{q}^{k}$ is the coefficient of mass transfer; $\alpha_{q}$ is the volume fraction of phase $q$, and the gas-liquid two phase is in state of laminar during the mass transport. In this simulation, the rate of nitrogen decomposition into nitrogen atoms is constant.

$$
\Gamma_{q}^{k}=D_{q}^{k} \rho_{q}
$$

Due to the heating, the phase transition and flow in molten pool occurred, which led to fluctuation at the phase interface. VOF method obtains the position of the phase interface by calculating and tracing the distribution of volume fraction $\alpha_{q}$. In a computing unit, when $\alpha_{q}=0$, phase $q$ is not shown in this unit; If $\alpha_{q}=1$, the phase $q$ is filled in this unit; when $0<\alpha_{\mathrm{q}}<1$, phase $q$ and other phases are contained in this unit. Therefore, the distribution of interface can be calculated by the following.

$$
\begin{gathered}
\frac{\partial \alpha_{q}}{\partial t}+\stackrel{r}{v} \nabla \alpha_{q}=0 \\
\sum_{q=L, G} \alpha_{q}=1
\end{gathered}
$$

The physical properties in the control equation are determined by each phase in the system. Thus, $\rho$ and $v$ in the gas-liquid two phase flow can be described by Eq. (11) (12).

$$
\begin{aligned}
& \rho=\alpha_{L} \rho_{L}+\left(1-\alpha_{L}\right) \rho_{G} \\
& \mathrm{v}=\alpha_{L} \rho_{L}+\left(1-\alpha_{L}\right) v_{G}
\end{aligned}
$$

The mass source $S_{G L}^{k}$ can be obtained based on the two film theory.

$$
S_{G L}^{k}=\left(m x_{k}-y_{k}\right)\left(\frac{m}{k_{L} \alpha_{e} M_{k} C_{L T}}+\frac{1}{k_{G} a_{e} M_{k} C_{G T}}\right)
$$

Where $x_{k}, y_{k}$ is the mole fraction of component $k$ in gas and liquid at phase interface. $\alpha_{e}$ is the effective mass ratio of the surface area $\left(m^{2}, m^{-\pi}\right), M_{k}$ is the relative atomic mass of the transfer element, and $C_{L T}$ and $C_{G T}$ are the mole concentrations in liquid and gas phases (kmol. $m^{-a}$ ).

$S_{G L}^{k}$ can be written using UDF in Fluent. Here, mass transfer conformed to penetration theory.

$$
k_{G, L}=2 \sqrt{\frac{D_{G, L}}{\pi T}}
$$

Where $T$ is the contact time, and $D_{\mathrm{L}} 、 D_{\mathrm{G}}$ are the diffusion coefficients of species $k$ in liquid and gas phases $\left(\mathrm{cm}^{2} / \mathrm{s}\right)$.

So the melting and solidification model, VOF model, the mass transfer model, and so on in Fluent were applied to simulate the process of laser nitriding. The source term of energy, mass transport, mass and momentum were written using in Fluent.

\section{Resulting and Discussion}

\subsection{The Temperature Field and Flow in Molten Pool}

From the numerical calculations, many details of the process were obtained. For the energy density was $8.5 \times 10^{8} \mathrm{~W} / \mathrm{m}^{2}$, the distributions of temperature field, the flow in molten pool, and the change in size of molten pool at different times are shown in Figure 2. This figure shows the temperature field (color scale), the flow direction of the melt (indicated by arrows), and the melt pool shape (surrounded by the red line) in a crosssectional view. The time-dependent development of a track during irradiation was calculated to be $5 \mathrm{~ms}$. The region of molten pool grew wider and deeper with continued irradiating.

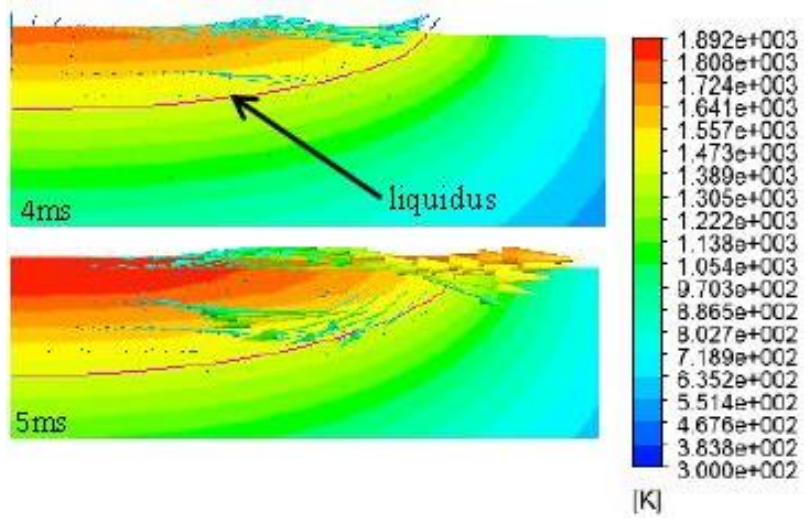

Fig. 2. Variation of molten pool shape and temperature field

The profiles of surface temperature versus time at different density are shown in Figure 3 . The temperature did not reach the boiling point in simulation because of the limiting treatment parameters (laser intensity and interaction time). Consequently, keyhole formation and evaporation could be avoided or minimized, which is a main requirement for cladding or alloying at laser material processing. With the sample is irradiated with the laser, the temperature on the surface increases as shown in Figure 3. Increasing the energy density caused the maximum temperature of the surface to increase and the flow in the pool to become accelerated. The 
temperature and the flow in the melting pool affect the behavior of heat transfer.

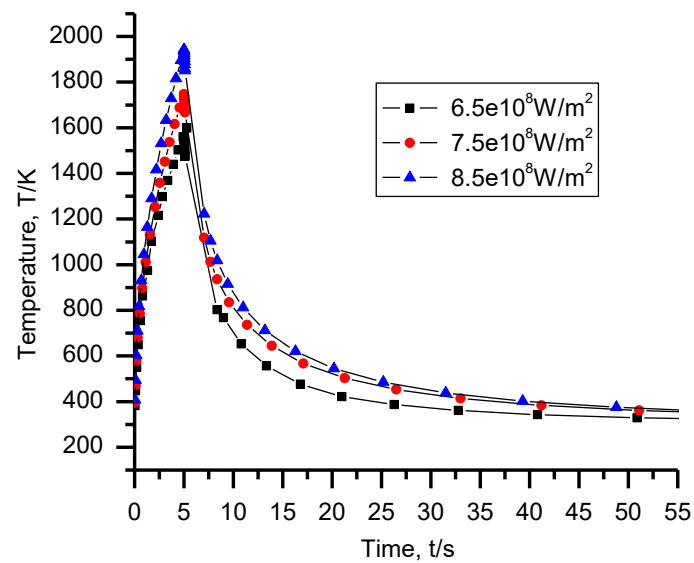

Fig. 3. variation of surface temperature with time

\subsection{The Concentration of Nitrogen in Melt Pool}

the higher temperature, the greater diffusion coefficient, means the nitrogen spread faster in uranium. At the same time, the greater gradient of the temperature provided thicker nitride layer. The pressure was at $0.5 \mathrm{MPa}$, and the energy density varied from $6.5 \times 10^{8} \mathrm{~W} / \mathrm{m}^{2}$ to $8.5 \times 10^{8}$ $\mathrm{W} / \mathrm{m}^{2}$; experimental and simulation results are shown Figure 4. At the same pressure as in ambient, the concentration of nitrogen in melt pool rose with increasing energy density. The trend of simulation agreed with the experiment. Meanwhile, the decomposition of nitrogen gas was assumed to be constant in the numerical simulation, and the nitrogen in uranium would form nitride. The correction coefficient was applied to correct the differences.

$$
[R]_{e}=\beta[R]_{S}
$$

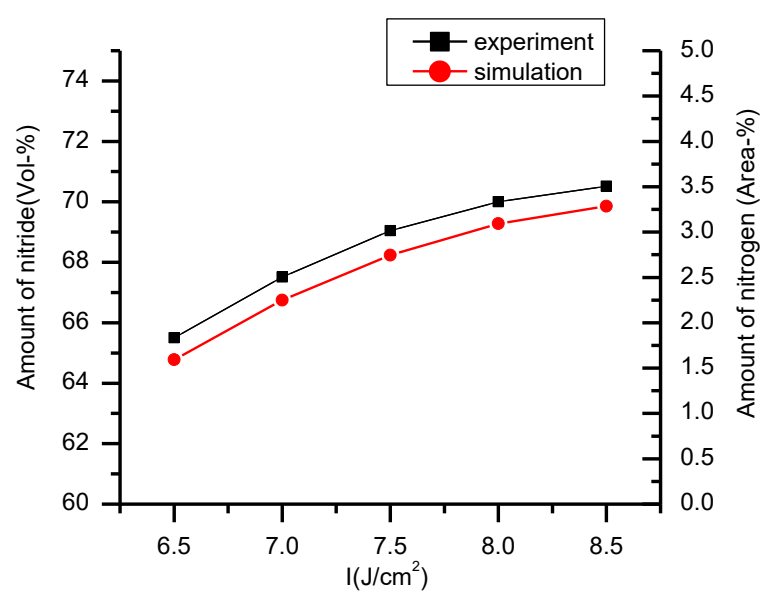

Fig. 4. Dependence of nitrogen concentration on nitrogen laser energy

According to the results of experiment and simulation above, the nitriding model is feasible. At the same time, the decomposition rate of nitrogen gas varied with temperature, and the nitrogen in the material will form nitride. All of these need to be further studied.
The influence factors on the diffusion and distribution of nitrogen can be acquired based on this nitriding model. The results were obtained to provide guides for production.

\subsection{The Distribution of Nitrogen}

Due to the strong convective flow, the nitrogen transport is a combination of diffusion and convection. Nitrogen surface profiles were measured for irradiated samples. The energy varied from $6.5 \times 10^{8} \mathrm{~W} / \mathrm{m}^{2}$ to $8.5 \times 10^{8} \mathrm{~W} / \mathrm{m}^{2}$ for one pulse. In Figure 5, when the energy is $6.5 \times 10^{8}$ $\mathrm{W} / \mathrm{m}^{2}$, only a small region in the central part is being nitrided. When the energy was $8.5 \times 10^{8} \mathrm{~W} / \mathrm{m}^{2}$, a region with broadest nitriding area appeared, covering the entire laser area. It was also observed that the edge of the nitriding region had higher concentration of nitrogen than the central region. It indicated that with the increasing of energy density, the temperature increased. Although the diffusive mass transport enhanced, the convective mass transport occupied the dominant position in the process of mass transport.

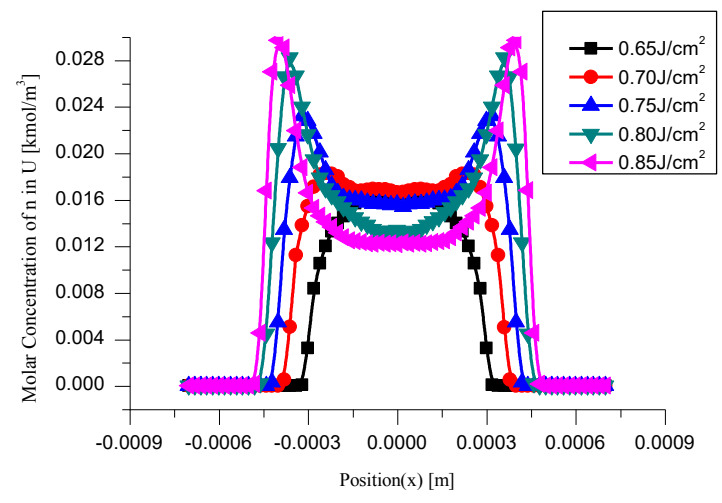

Fig. 4. Distribution of nitrogen at surface

\section{Conclusion}

This paper established a numerical model for simulating the laser nitriding of uranium in an ambient nitrogen atmosphere. The result showed that the energy density of the laser had the main influence on the temperature field of laser nitriding. The higher the laser density, the greater the gradient of temperature; it led to strong Marangoni convection in the molten pool. Due to the supplemental convective, the aspect ratio of the melt pool was very high. However, the higher temperature would lead to decrease in solidification rate. As a result, a coarse solidification microstructure would be developed.

The density of energy not only determines the size of nitriding area, but also determines the distribution of nitrogen in the molten pool; it affects the formation of the nitride layer. The higher energy density would not lead to ideal distribution of nitrogen at the surface. Meanwhile, the lower energy density only provided a narrow nitriding region. In order to get the uniform nitriding layer, reasonable laser parameters should be taken into consideration. In this simulation, the ideal 
threshold laser energy is around $7.0 \times 10^{8} \mathrm{~W} / \mathrm{m}^{2}$. The calculated results are consistent with the experiments.

\section{References}

1. D. M. Mattox, R. D. Bland. Journal of Nuclear Materials 21, 3 (1967):349-352.

2. R. Arkush, M. H. Mintz, N Shamir, \& R Arkush. Journal of Nuclear Material 281, 2(2000): 182-190.

3. Peter Schaaf. Progress in Materials Science 47, (2002): 1-161.
4. D. Höche, S. Müller, G. Rapin, M. Shinn, E. Remdt \& M. Gubisch et al. Metallurgical \& Materials Transactions B 40, 4(2009): 497-507.

5. Höche D, Rapin G \& Schaaf P. Applied Surface Science 254, 4(2007): 888-892.

6. FuZhen, Xuan, LiQin, Cao, Zhengdong, Wang et al. Computational Materials Science 49, 1(2010):104111.

7. Yongbin Zhang, Daqiao Meng Youshou Zhang. Journal of Nuclear Materials 397, (2010): 31-35.

8. ZhiLei Chen, Yongbin Zhang. Acta Phys. Chim.Sin. (Suppl.), 2015, 0001-0009. 